\title{
Sufficient conditions for analytic functions defined by Frasin differential operator
}

\author{
Tariq Al-Hawary
}

Abstract. Very recently, Frasin [7] introduced the differential operator $\mathcal{I}_{m, \lambda}^{\zeta} f(z)$ defined as

$$
\mathcal{I}_{m, \lambda}^{\zeta} f(z)=z+\sum_{n=2}^{\infty}\left(1+(n-1) \sum_{j=1}^{m}\left(\begin{array}{c}
m \\
j
\end{array}\right)(-1)^{j+1} \lambda^{j}\right)^{\zeta} a_{n} z^{n} .
$$

The current work contributes to give an application of the differential operator $\mathcal{I}_{m, \lambda}^{\zeta} f(z)$ to the differential inequalities in the complex plane.

Mathematics Subject Classification (2010): 30C45.

Keywords: Analytic functions, differential operator.

\section{Introduction and preliminaries}

Let $\mathcal{A}$ be the class of all normalized analytic functions in $\mathbb{U}=\{z \in \mathbb{C}:|z|<1\}$ that has a Taylor-Maclaurin series expansion of the form:

$$
f(z)=z+\sum_{n=2}^{\infty} a_{n} z^{n}
$$

For a function $f$ in $\mathcal{A}$, and using the binomial series

$$
(1-\lambda)^{m}=\sum_{j=0}^{m}\left(\begin{array}{c}
m \\
j
\end{array}\right)(-1)^{j} \lambda^{j} \quad\left(m \in \mathbb{N}, j \in \mathbb{N}_{0}=\mathbb{N} \cup\{0\}, \mathbb{N}=\{1,2, \cdots\}\right),
$$

let $\mathcal{I}_{m, \lambda}^{\zeta} f(z)$ be the differential operator defined as follows:

$$
\begin{aligned}
\mathcal{I}^{0} f(z) & =f(z), \\
\mathcal{I}_{m, \lambda}^{1} f(z) & =(1-\lambda)^{m} f(z)+\left(1-(1-\lambda)^{m}\right) z f^{\prime}(z)=\mathcal{I}_{m, \lambda} f(z), \lambda>0 ; m \in \mathbb{N}, \\
\mathcal{I}_{m, \lambda}^{\zeta} f(z) & =\mathcal{I}_{m, \lambda}\left(\mathcal{I}^{\zeta-1} f(z)\right) \quad(\zeta \in \mathbb{N}) .
\end{aligned}
$$


For $f \in \mathcal{A}$, we see that

$$
\mathcal{I}_{m, \lambda}^{\zeta} f(z)=z+\sum_{n=2}^{\infty}\left(1+(n-1) \sum_{j=1}^{m}\left(\begin{array}{c}
m \\
j
\end{array}\right)(-1)^{j+1} \lambda^{j}\right)^{\zeta} a_{n} z^{n}, \zeta \in \mathbb{N}_{0} .
$$

Using (1.3), it is easily verified that

$$
C_{j}^{m}(\lambda) z\left(\mathcal{I}_{m, \lambda}^{\zeta} f(z)\right)^{\prime}=\mathcal{I}_{m, \lambda}^{\zeta+1} f(z)-\left(1-C_{j}^{m}(\lambda)\right) \mathcal{I}_{m, \lambda}^{\zeta} f(z), \quad \zeta \in \mathbb{N}_{0},
$$

where $C_{j}^{m}(\lambda):=\sum_{j=1}^{m}\left(\begin{array}{c}m \\ j\end{array}\right)(-1)^{j+1} \lambda^{j}$.

From the identity (1.4), we readily have

$$
C_{j}^{m}(\lambda) z\left(\mathcal{I}_{m, \lambda}^{\zeta-1} f(z)\right)^{\prime}=\mathcal{I}_{m, \lambda}^{\zeta} f(z)-\left(1-C_{j}^{m}(\lambda)\right) \mathcal{I}_{m, \lambda}^{\zeta-1} f(z), \quad \zeta \in \mathbb{N}_{0}
$$

and

$$
C_{j}^{m}(\lambda) z\left(\mathcal{I}_{m, \lambda}^{\zeta+1} f(z)\right)^{\prime}=\mathcal{I}_{m, \lambda}^{\zeta+2} f(z)-\left(1-C_{j}^{m}(\lambda)\right) \mathcal{I}_{m, \lambda}^{\zeta+1} f(z), \quad \zeta \in \mathbb{N}_{0} .
$$

The above differential operator $\mathcal{I}_{m, \lambda}^{\zeta} f(z)$ was introduced and studied by Frasin [7]. Note that for $m=1$, we obtain the differential operator $\mathcal{I}_{1, \lambda}^{\zeta}$ defined by Al-Oboudi [1] and for $m=\lambda=1$, we get Sălăgean differential operator $\mathcal{I}^{\zeta}[9]$ (see also Aouf [2,3]). Our aim in this work is to provide an application of the differential operator $\mathcal{I}_{m, \lambda}^{\zeta} f(z)$, (see for example, $[4,5,6,8,10]$ ).

For our purpose, using the operator $\mathcal{I}_{m, \lambda}^{\zeta} f(z)$, we define the classes $Q$ and $G$ respectively.

Definition 1.1. Let $Q$ be the set of continuous complex functions $q(r, s, t): \mathbb{C}^{3} \rightarrow \mathbb{C}$ in $\mathbb{D} \subset \mathbb{C}^{3}$ such that $(0,0,0) \in \mathbb{D},|q(0,0,0)|<1$ and

$$
\begin{aligned}
& \mid q\left(e^{i \theta},\left[C_{j}^{m}(\lambda) \delta+\left(1-C_{j}^{m}(\lambda)\right)\right] e^{i \theta},\right. \\
& {\left[C_{j}^{m}(\lambda)\right]^{2} \beta+\left[C_{j}^{m}(\lambda)\left(2-C_{j}^{m}(\lambda)\right) \delta+\left(1-C_{j}^{m}(\lambda)\right)^{2}\right] e^{i \theta} \mid } \\
\geq & 1
\end{aligned}
$$

whenever

$$
\begin{aligned}
&\left(e^{i \theta},\left[C_{j}^{m}(\lambda) \delta+\left(1-C_{j}^{m}(\lambda)\right)\right] e^{i \theta},\right. \\
& {\left.\left[C_{j}^{m}(\lambda)\right]^{2} \beta+\left[C_{j}^{m}(\lambda)\left(2-C_{j}^{m}(\lambda)\right) \delta+\left(1-C_{j}^{m}(\lambda)\right)^{2}\right] e^{i \theta}\right) } \\
& \in \mathbb{D}
\end{aligned}
$$

with $\operatorname{Re}\left\{\beta e^{-i \theta}\right\} \geq \delta(\delta-1)$ for real $\theta, \delta \geq 1$.

Definition 1.2. Let $G$ be the set of continuous complex functions $g(r, s, t): \mathbb{C}^{3} \rightarrow \mathbb{C}$ in $\mathbb{D} \subset \mathbb{C}^{3}$ such that $(1,1,1) \in \mathbb{D},|g(1,1,1)|<L \quad(L>1)$ and

$$
\left|g\left(L e^{i \theta}, L e^{i \theta}+C_{j}^{m}(\lambda) \delta, \frac{\left[C_{j}^{m}(\lambda)\right]^{2}(\delta+\mu)+3 L C_{j}^{m}(\lambda) \delta e^{i \theta}+L^{2} e^{2 i \theta}}{C_{j}^{m}(\lambda)\left(L e^{i \theta}+C_{j}^{m}(\lambda) \delta\right)}\right)\right| \geq L
$$

whenever

$$
\left(L e^{i \theta}, L e^{i \theta}+C_{j}^{m}(\lambda) \delta, \frac{\left[C_{j}^{m}(\lambda)\right]^{2}(\delta+\mu)+3 L C_{j}^{m}(\lambda) \delta e^{i \theta}+L^{2} e^{2 i \theta}}{C_{j}^{m}(\lambda)\left(L e^{i \theta}+C_{j}^{m}(\lambda) \delta\right)}\right) \in \mathbb{D}
$$


with $\operatorname{Re}\{\mu\} \geq \delta(\delta-1)$ for real $\theta, \delta \geq \frac{L-1}{L+1}$.

\section{Main results}

To prove our theorems in this section, we recall two lemmas for Miller and Mocanu.

Lemma 2.1. [8] Let a function $w(z) \in \mathcal{A}$ with $w(z) \neq 0$ in $\mathbb{U}$. If $z_{0}=r_{0} e^{i \theta}\left(0<r_{0}<1\right)$ and $\left|w\left(z_{0}\right)\right|=\max _{|z| \leq r_{0}}|w(z)|$. Then

$$
z_{0} w^{\prime}\left(z_{0}\right)=\delta w\left(z_{0}\right)
$$

and

$$
\operatorname{Re}\left\{1+\frac{z_{0} w^{\prime \prime}\left(z_{0}\right)}{w^{\prime}\left(z_{0}\right)}\right\} \geq \delta, \quad \delta \geq 1 .
$$

Lemma 2.2. [8] Let $w(z)=a+w_{k} z^{k}+\cdots$ be analytic in $\mathbb{U}$ with $w(z) \neq a$ and $k \geq 1$. If $z_{0}=r_{0} e^{i \theta}\left(0<r_{0}<1\right)$ and $\left|w\left(z_{0}\right)\right|=\max _{|z| \leq r_{0}}|w(z)|$. Then

$$
z_{0} w^{\prime}\left(z_{0}\right)=\delta w\left(z_{0}\right)
$$

and

$$
\operatorname{Re}\left\{1+\frac{z_{0} w^{\prime \prime}\left(z_{0}\right)}{w^{\prime}\left(z_{0}\right)}\right\} \geq \delta, \quad(\delta \in \mathbb{R})
$$

where

$$
\delta \geq k \frac{\left|w\left(z_{0}\right)-a\right|^{2}}{\left|w\left(z_{0}\right)\right|^{2}-|a|^{2}} \geq k \frac{\left|w\left(z_{0}\right)\right|-|a|}{\left|w\left(z_{0}\right)\right|+|a|} .
$$

Applying Lemma 2.1, we prove Theorem 2.3.

Theorem 2.3. Let $q(r, s, t) \in Q$ and $f(z) \in \mathcal{A}$ such that

$$
\left(\mathcal{I}_{m, \lambda}^{\zeta} f(z), \mathcal{I}_{m, \lambda}^{\zeta+1} f(z), \mathcal{I}_{m, \lambda}^{\zeta+2} f(z)\right) \in \mathbb{D} \subset \mathbb{C}^{3}
$$

and

$$
\left|q\left(\mathcal{I}_{m, \lambda}^{\zeta} f(z), \mathcal{I}_{m, \lambda}^{\zeta+1} f(z), \mathcal{I}_{m, \lambda}^{\zeta+2} f(z)\right)\right|<1
$$

for $\zeta \in \mathbb{N}_{0}, m \in \mathbb{N}, \lambda>0$ and $z \in \mathbb{U}$. Then

$$
\left|\mathcal{I}_{m, \lambda}^{\zeta} f(z)\right|<1 \quad(z \in \mathbb{U})
$$

Proof. Let

$$
\mathcal{I}_{m, \lambda}^{\zeta} f(z)=w(z)
$$

then $w(z) \in \mathcal{A}$ and $w(z) \neq 0(z \in \mathbb{U})$. Using the identity (1.4), we have

$$
\mathcal{I}_{m, \lambda}^{\zeta+1} f(z)=C_{j}^{m}(\lambda) z w^{\prime}(z)+\left(1-C_{j}^{m}(\lambda)\right) w(z)
$$

and

$$
\mathcal{I}_{m, \lambda}^{\zeta+2} f(z)=\left[C_{j}^{m}(\lambda)\right]^{2}\left(z^{2} w^{\prime \prime}(z)\right)+C_{j}^{m}(\lambda)\left(2-C_{j}^{m}(\lambda)\right) z w^{\prime}(z)+\left(1-C_{j}^{m}(\lambda)\right)^{2} w(z) .
$$


Letting $z_{0}=r_{0} e^{i \theta}\left(0<r_{0}<1\right),\left|w\left(z_{0}\right)\right|=\max _{|z| \leq r_{0}}|w(z)|=1, w\left(z_{0}\right)=e^{i \theta}$ and using (2.1), we have

$$
\begin{aligned}
\mathcal{I}_{m, \lambda}^{\zeta} f\left(z_{0}\right) & =w\left(z_{0}\right)=e^{i \theta} \\
\mathcal{I}_{m, \lambda}^{\zeta+1} f\left(z_{0}\right) & =C_{j}^{m}(\lambda) \delta w\left(z_{0}\right)+\left(1-C_{j}^{m}(\lambda)\right) w\left(z_{0}\right) \\
& =\left[C_{j}^{m}(\lambda) \delta+\left(1-C_{j}^{m}(\lambda)\right)\right] e^{i \theta}
\end{aligned}
$$

and

$$
\begin{aligned}
\mathcal{I}_{m, \lambda}^{\zeta+2} f\left(z_{0}\right) & =\left[C_{j}^{m}(\lambda)\right]^{2}\left(z_{0}^{2} w^{\prime \prime}\left(z_{0}\right)\right)+C_{j}^{m}(\lambda)\left(2-C_{j}^{m}(\lambda)\right) \delta w\left(z_{0}\right)+\left(1-C_{j}^{m}(\lambda)\right)^{2} w\left(z_{0}\right) \\
& =\left[C_{j}^{m}(\lambda)\right]^{2} \beta+\left[C_{j}^{m}(\lambda)\left(2-C_{j}^{m}(\lambda)\right) \delta+\left(1-C_{j}^{m}(\lambda)\right)^{2}\right] e^{i \theta} .
\end{aligned}
$$

where

$$
\beta=z_{0}^{2} w^{\prime \prime}\left(z_{0}\right) \quad \text { and } \quad \delta \geq 1 .
$$

Moreover, an application of (2.2) gives

$$
\operatorname{Re}\left\{\frac{z_{0} w^{\prime \prime}\left(z_{0}\right)}{w^{\prime}\left(z_{0}\right)}\right\}=\operatorname{Re}\left\{\frac{z_{0}^{2} w^{\prime \prime}\left(z_{0}\right)}{\delta e^{i \theta}}\right\} \geq \delta-1,
$$

or

$$
\operatorname{Re}\left\{\beta e^{-i \theta}\right\} \geq \delta(\delta-1)
$$

Since $q(r, s, t) \in Q$, we have

$$
\begin{aligned}
& \left|q\left(\mathcal{I}_{m, \lambda}^{\zeta} f(z), \mathcal{I}_{m, \lambda}^{\zeta+1} f(z), \mathcal{I}_{m, \lambda}^{\zeta+2} f(z)\right)\right| \\
= & \mid q\left(e^{i \theta},\left[C_{j}^{m}(\lambda) \delta+\left(1-C_{j}^{m}(\lambda)\right)\right] e^{i \theta},\right. \\
& {\left.\left[C_{j}^{m}(\lambda)\right]^{2} \beta+\left[C_{j}^{m}(\lambda)\left(2-C_{j}^{m}(\lambda)\right) \delta+\left(1-C_{j}^{m}(\lambda)\right)^{2}\right] e^{i \theta}\right) \mid } \\
> & 1
\end{aligned}
$$

which opposes the condition (2.6) of Theorem 2.3. So we have

$$
\left|\mathcal{I}_{m, \lambda}^{\zeta} f(z)\right|<1 \quad(z \in \mathbb{U})
$$

In Theorem 2.3, if $\zeta=0, \lambda=1$ and $m=1$ we get

Corollary 2.4. Let $q(r, s, t) \in Q$ and $f(z) \in \mathcal{A}$ such that

$$
\left(f(z), z f^{\prime}(z), z^{2} f^{\prime \prime}(z)+z f^{\prime}(z)\right) \in \mathbb{D} \subset \mathbb{C}^{3}
$$

and

$$
\left|q\left(f(z), z f^{\prime}(z), z^{2} f^{\prime \prime}(z)+z f^{\prime}(z)\right)\right|<1, \quad z \in \mathbb{U} .
$$

Then

$$
|f(z)|<1 \quad(z \in \mathbb{U}) .
$$

Now, using Lemma 2.2 we will prove the following theorem. 
Theorem 2.5. Let $g(r, s, t) \in G$ and $f(z) \in \mathcal{A}$ satisfy

$$
\left(\frac{\mathcal{I}_{m, \lambda}^{\zeta} f(z)}{\mathcal{I}_{m, \lambda}^{\zeta-1} f(z)}, \frac{\mathcal{I}_{m, \lambda}^{\zeta+1} f(z)}{\mathcal{I}_{m, \lambda}^{\zeta} f(z)}, \frac{\mathcal{I}_{m, \lambda}^{\zeta+2} f(z)}{\mathcal{I}_{m, \lambda}^{\zeta+1} f(z)}\right) \in \mathbb{D} \subset \mathbb{C}^{3}
$$

and

$$
\left|g\left(\frac{\mathcal{I}_{m, \lambda}^{\zeta} f(z)}{\mathcal{I}_{m, \lambda}^{\zeta-1} f(z)}, \frac{\mathcal{I}_{m, \lambda}^{\zeta+1} f(z)}{\mathcal{I}_{m, \lambda}^{\zeta} f(z)}, \frac{\mathcal{I}_{m, \lambda}^{\zeta+2} f(z)}{\mathcal{I}_{m, \lambda}^{\zeta+1} f(z)}\right)\right|<L
$$

for $m \in \mathbb{N}, \zeta \geq 1, \lambda>0, L>1$ and all $z \in \mathbb{U}$. Then

$$
\left|\frac{\mathcal{I}_{m, \lambda}^{\zeta} f(z)}{\mathcal{I}_{m, \lambda}^{\zeta-1} f(z)}\right|<L \quad(z \in \mathbb{U})
$$

Proof. Let

$$
\frac{\mathcal{I}_{m, \lambda}^{\zeta} f(z)}{\mathcal{I}_{m, \lambda}^{\zeta-1} f(z)}=w(z) \quad(\zeta \geq 1)
$$

Then $w(z)$ is analytic function in $\mathbb{U}, w(0)=1$ and $w(z) \neq 1$. Differentiating $(2.10)$ logarithmically and multiplying by $z$, we get

$$
\frac{z\left(\mathcal{I}_{m, \lambda}^{\zeta} f(z)\right)^{\prime}}{\mathcal{I}_{m, \lambda}^{\zeta} f(z)}-\frac{z\left(\mathcal{I}_{m, \lambda}^{\zeta-1} f(z)\right)^{\prime}}{\mathcal{I}_{m, \lambda}^{\zeta-1} f(z)}=\frac{z w^{\prime}(z)}{w(z)} .
$$

Using the identities (1.4) and (1.5), we have

$$
\frac{\mathcal{I}_{m, \lambda}^{\zeta+1} f(z)}{\mathcal{I}_{m, \lambda}^{\zeta} f(z)}=w(z)+C_{j}^{m}(\lambda) \frac{z w^{\prime}(z)}{w(z)} .
$$

Differentiating (2.11) logarithmically and multiply by $z$, we have

$$
\begin{aligned}
& \frac{z\left(\mathcal{I}_{m, \lambda}^{\zeta+1} f(z)\right)^{\prime}}{\mathcal{I}_{m, \lambda}^{\zeta+1} f(z)}-\frac{z\left(\mathcal{I}_{m, \lambda}^{\zeta} f(z)\right)^{\prime}}{\mathcal{I}_{m, \lambda}^{\zeta} f(z)} \\
= & \frac{z\left[w(z)+C_{j}^{m}(\lambda) \frac{z w^{\prime}(z)}{w(z)}\right]^{\prime}}{w(z)+C_{j}^{m}(\lambda) \frac{z w^{\prime}(z)}{w(z)}} \\
= & \frac{z w^{\prime}(z)+C_{j}^{m}(\lambda)\left[\frac{z w^{\prime}(z)}{w(z)}+\frac{z^{2} w^{\prime \prime}(z)}{w(z)}-\left(\frac{z w^{\prime}(z)}{w(z)}\right)^{2}\right]}{w(z)+C_{j}^{m}(\lambda) \frac{z w^{\prime}(z)}{w(z)}} .
\end{aligned}
$$


Using the identities (1.4) and (1.6), it follows from (2.12) that

$$
\begin{aligned}
& \frac{1}{C_{j}^{m}(\lambda)} \frac{\mathcal{I}_{m, \lambda}^{\zeta+2} f(z)}{\mathcal{I}_{m, \lambda}^{\zeta+1} f(z)} \\
= & \frac{1}{C_{j}^{m}(\lambda)} \frac{\mathcal{I}_{m, \lambda}^{\zeta+1} f(z)}{\mathcal{I}_{m, \lambda}^{\zeta} f(z)}+\frac{z w^{\prime}(z)+C_{j}^{m}(\lambda)\left[\frac{z w^{\prime}(z)}{w(z)}+\frac{z^{2} w^{\prime \prime}(z)}{w(z)}-\left(\frac{z w^{\prime}(z)}{w(z)}\right)^{2}\right]}{w(z)+C_{j}^{m}(\lambda) \frac{z w^{\prime}(z)}{w(z)}} \\
= & \frac{1}{C_{j}^{m}(\lambda)} w(z)+\frac{z w^{\prime}(z)}{w(z)}+\frac{z w^{\prime}(z)+C_{j}^{m}(\lambda)\left[\frac{z w^{\prime}(z)}{w(z)}+\frac{z^{2} w^{\prime \prime}(z)}{w(z)}-\left(\frac{z w^{\prime}(z)}{w(z)}\right)^{2}\right]}{w(z)+C_{j}^{m}(\lambda) \frac{z w^{\prime}(z)}{w(z)}}
\end{aligned}
$$

Letting $z_{0}=r_{0} e^{i \theta}\left(0<r_{0}<1\right), \max _{|z| \leq r_{0}}|w(z)|=\left|w\left(z_{0}\right)\right|=L, w\left(z_{0}\right)=L e^{i \theta}$ and using Lemma 2.2 with $a=1$ and $k=1$, we have

$$
\begin{aligned}
& \frac{\mathcal{I}_{m, \lambda}^{\zeta} f\left(z_{0}\right)}{\mathcal{I}_{m, \lambda}^{\zeta-1} f\left(z_{0}\right)}=L e^{i \theta}, \\
& \frac{\mathcal{I}_{m, \lambda}^{\zeta+1} f\left(z_{0}\right)}{\mathcal{I}_{m, \lambda}^{\zeta} f\left(z_{0}\right)}=L e^{i \theta}+C_{j}^{m}(\lambda) \delta, \\
& \frac{\mathcal{I}_{m, \lambda}^{\zeta+2} f\left(z_{0}\right)}{\mathcal{I}_{m, \lambda}^{\zeta+1} f\left(z_{0}\right)}=\frac{\left[C_{j}^{m}(\lambda)\right]^{2}(\delta+\mu)+3 L C_{j}^{m}(\lambda) \delta e^{i \theta}+L^{2} e^{2 i \theta}}{C_{j}^{m}(\lambda)\left(L e^{i \theta}+C_{j}^{m}(\lambda) \delta\right)},
\end{aligned}
$$

where

$$
\mu=\frac{z_{0}^{2} w^{\prime \prime}\left(z_{0}\right)}{w\left(z_{0}\right)} \quad \text { and } \quad \delta \geq \frac{L-1}{L+1} .
$$

Moreover, an application of (2.2) gives $\operatorname{Re}\{\mu\} \geq \delta(\delta-1)$.

Since $g(r, s, t) \in G$, we have

$$
\begin{aligned}
& \left|g\left(\frac{\mathcal{I}_{m, \lambda}^{\zeta} f\left(z_{0}\right)}{\mathcal{I}_{m, \lambda}^{\zeta-1} f\left(z_{0}\right)}, \frac{\mathcal{I}_{m, \lambda}^{\zeta+1} f\left(z_{0}\right)}{\mathcal{I}_{m, \lambda}^{\zeta} f\left(z_{0}\right)}, \frac{\mathcal{I}_{m, \lambda}^{\zeta+2} f\left(z_{0}\right)}{\mathcal{I}_{m, \lambda}^{\zeta+1} f\left(z_{0}\right)}\right)\right| \\
= & \left|g\left(L e^{i \theta}, L e^{i \theta}+C_{j}^{m}(\lambda) \delta, \frac{\left[C_{j}^{m}(\lambda)\right]^{2}(\delta+\mu)+3 L C_{j}^{m}(\lambda) \delta e^{i \theta}+L^{2} e^{2 i \theta}}{C_{j}^{m}(\lambda)\left(L e^{i \theta}+C_{j}^{m}(\lambda) \delta\right)}\right)\right| \\
\geq & L
\end{aligned}
$$

which contradicts the condition (2.9) of Theorem 2.5. Thus

$$
|w(z)|=\left|\frac{\mathcal{I}_{m, \lambda}^{\zeta} f(z)}{\mathcal{I}_{m, \lambda}^{\zeta-1} f(z)}\right|<L .
$$

for $m \in \mathbb{N}, \zeta \geq 1, \lambda>0$ and all $z \in \mathbb{U}$. The proof is complete.

In Theorem 2.5 , if $\zeta=1, \lambda=1$ and $m=1$ we get 
Corollary 2.6. Let $g(r, s, t) \in G$ and $f(z) \in \mathcal{A}$ satisfy

$$
\left(\frac{z f^{\prime}(z)}{f(z)}, \frac{z f^{\prime \prime}(z)+f^{\prime}(z)}{f^{\prime}(z)}, \frac{z^{2} f^{(3)}(z)+3 z f^{\prime \prime}(z)+f^{\prime}(z)}{z f^{\prime \prime}(z)+f^{\prime}(z)}\right) \in \mathbb{D} \subset \mathbb{C}^{3}
$$

and

$$
\left|g\left(\frac{z f^{\prime}(z)}{f(z)}, \frac{z f^{\prime \prime}(z)+f^{\prime}(z)}{f^{\prime}(z)}, \frac{z^{2} f^{(3)}(z)+3 z f^{\prime \prime}(z)+f^{\prime}(z)}{z f^{\prime \prime}(z)+f^{\prime}(z)}\right)\right|<L
$$

for $L>1$ and all $z \in \mathbb{U}$. Then

$$
\left|\frac{z f^{\prime}(z)}{f(z)}\right|<L \quad(z \in \mathbb{U})
$$

\section{References}

[1] Al-Oboudi, F.M., On univalent functions defined by a generalized Sălăgean operator, Int. J. Math. Math. Sci., (2004), no.25-28, 1429-1436.

[2] Aouf, M.K., Neighorhoods of certain classes of analytic functions with negative coeffcients, Internat. J. Math., Vol. 2006, Art. ID 38258, 1-6.

[3] Aouf, M.K., On certain multivalent functions with negative coefficients defined by using a differential operator, Mat. Vesnik, 62(2010), no. 1, 23-35.

[4] Aouf, M.K., Hossen, H.M., Lashin, A.Y., An application of certain integral operators, J. Math. Anal. Appl., 248(2000), 475-481.

[5] El-Ashwah, R.M., Aouf, M.K., Some properties of new integral operator, Acta Universitatis Apulensis, 24(2010), 51-61.

[6] El-Ashwah, R.M., Aouf, M.K., Bulboacă, T., An application of generalized integral operator, Stud. Univ. Babeş-Bolyai Math., 57(2012), no. 2, 189-193.

[7] Frasin, B.A., A new differential operator of analytic functions involving binomial series, Bol. Soc. Paran. Mat. (in press).

[8] Miller, S.S., Mocanu, P.T., Second order differential inequalities in the complex plane, J. Math. Anal. Appl., 65(1978), 289-305.

[9] Sălăgean, G., Subclasses of univalent functions, in "Complex Analysis: Fifth Romanian - Finnish Seminar", Part I (Bucharest, 1981), 362-372, Lecture Notes in Mathematics, Vol. 1013, Springer-Verlag, Berlin/New York, 1983.

[10] Yousef, F., Al-Hawary, T., Murugusundaramoorthy, G., Fekete-Szegö functional problems for some subclasses of bi-univalent functions defined by Frasin differential operator, Afrika Matematika, (2019), 1-9.

Tariq Al-Hawary

Al-Balqa Applied University,

Department of Applied Science,

Ajloun College,

26816 Ajloun, Jordan

e-mail: tariq_amh@bau.edu.jo 